\title{
MICRORNAS AS BIOMARKERS FOR BREAST CANCER PROGNOSIS: A SYSTEMATIC REVIEW
}

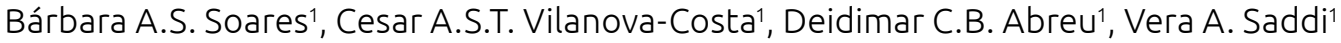 \\ ${ }^{1}$ Pontifícia Universidade Católica de Goiás, Associação de Combate ao Câncer em Goiás - Goiânia (GO), Brazil.
}

Objectives: Breast cancer is an important health problem worldwide and the identification of prognostic markers in breast cancer is important in order to establish the most successful treatment for each patient. MiRNAs are non-coding RNAs, which modulate the expression of more than $50 \%$ of human genes at the post-transcriptional level. Deregulation of miRs is described in many types of tumors leading to their acting as tumor suppressors or oncogenes. This systematic review of the literature aimed to highlight tumor miRNA quantification, by real time PCR, as prognostic biomarkers in breast cancer. Methods: We systematically searched the PubMed database, in order to identify eligible studies and 1457 articles were initially selected. After reading the abstracts, 74 articles were read in complete and 20 studies were included in the review. Results: Twenty studies investigating the association between tumoral miRNA expression levels (predictive factors) and the prognostic in breast cancer patients were included in the review. MiRNA-21 and miRNA-200b were the most commonly investigated miRNA in breast cancer prognosis. Lymph node metastasis was significantly associated with the overexpression of miRNA-21, miRNA-301 and miRNA-370, and also to the under expression of miRNA-124, miRNA-127, miRNA-129-5p, miRNA199-5p, miRNA-206, miRNA-218, and miRNA-339-5. Distant metastasis was associated to miRNA-204 under expression. Tumor size was associated with the overexpression of miRNA-21 and miRNA-301, and also to under expression of miRNA-29b and miRNA129-5p. In relation to prognosis, lower survival rates were associated with the overexpression of miRNA-21, miRNA-301 and microRNA- 711, and with under expression of miRNA-15a, miRNA-29b, miRNA-124, miRNA-129-5p, miRNA-199b-5p, miRNA-200b, miRNA-204, miRNA-206 and miRNA-218. Higher survival rates were associated with the overexpression of miRNA-339-5 and miRNA-127 On the other hand, higher survival rates were associated with overexpression of miRNA-339-5 and miRNA-127, and also to under expression of miRNA-210. Conclusion: According to the results, preclinical and clinical investigation performed on tissue-specific miRNAs can be considered as novel promising biomarkers for prediction of prognosis in breast cancer patients. 